\title{
Changes in the gut microbiome and fermentation products concurrent with enhanced longevity in acarbose-treated mice
}

Byron J Smith ${ }^{1}$, Richard A Miller ${ }^{2}$, Aaron C Ericsson ${ }^{3}$, David C Harrison ${ }^{4}$, Randy Strong 5,6,7

and Thomas M Schmidt ${ }^{1,8^{*}}$ (D)

\begin{abstract}
Background: Treatment with the $\alpha$-glucosidase inhibitor acarbose increases median lifespan by approximately 20\% in male mice and $5 \%$ in females. This longevity extension differs from dietary restriction based on a number of features, including the relatively small effects on weight and the sex-specificity of the lifespan effect. By inhibiting host digestion, acarbose increases the flux of starch to the lower digestive system, resulting in changes to the gut microbiota and their fermentation products. Given the documented health benefits of short-chain fatty acids (SCFAs), the dominant products of starch fermentation by gut bacteria, this secondary effect of acarbose could contribute to increased longevity in mice. To explore this hypothesis, we compared the fecal microbiome of mice treated with acarbose to control mice at three independent study sites.
\end{abstract}

Results: Microbial communities and the concentrations of SCFAs in the feces of mice treated with acarbose were notably different from those of control mice. At all three study sites, the bloom of a single bacterial taxon was the most obvious response to acarbose treatment. The blooming populations were classified to the largely uncultured Bacteroidales family Muribaculaceae and were the same taxonomic unit at two of the three sites. Propionate concentrations in feces were consistently elevated in treated mice, while the concentrations of acetate and butyrate reflected a dependence on study site. Across all samples, Muribaculaceae abundance was strongly correlated with propionate and community composition was an important predictor of SCFA concentrations. Cox proportional hazards regression showed that the fecal concentrations of acetate, butyrate, and propionate were, together, predictive of mouse longevity even while controlling for sex, site, and acarbose.

Conclusion: We observed a correlation between fecal SCFAs and lifespan in mice, suggesting a role of the gut microbiota in the longevity-enhancing properties of acarbose. Treatment modulated the taxonomic composition and fermentation products of the gut microbiome, while the site-dependence of the responses illustrate the challenges facing reproducibility and interpretation in microbiome studies. These results motivate future studies exploring manipulation of the gut microbial community and its fermentation products for increased longevity, testing causal roles of SCFAs in the observed effects of acarbose.

Keywords: Acarbose, Longevity, Gut microbiome, Short-chain fatty acids, Muribaculaceae, S24-7, Candidatus Homeothermaceae

\footnotetext{
*Correspondence: schmidti@umich.edu

'Department of Ecology \& Evolutionary Biology, University of Michigan, 48109 Ann Arbor, MI, USA

${ }^{8}$ Department of Internal Medicine, University of Michigan, 48109 Ann Arbor,

MI, USA
}

Full list of author information is available at the end of the article

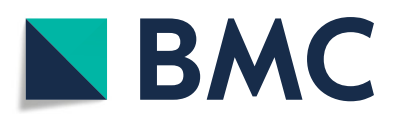

(c) The Author(s). 2019 Open Access This article is distributed under the terms of the Creative Commons Attribution 4.0 International License (http://creativecommons.org/licenses/by/4.0/), which permits unrestricted use, distribution, and reproduction in any medium, provided you give appropriate credit to the original author(s) and the source, provide a link to the Creative Commons license, and indicate if changes were made. The Creative Commons Public Domain Dedication waiver (http://creativecommons.org/publicdomain/zero/1.0/) applies to the data made available in this article, unless otherwise stated. 


\section{Background}

The Interventions Testing Program (ITP) is a longrunning, well-powered study of longevity enhancing interventions in genetically heterogeneous mice with identical protocols replicated at each of three study sites [1]. The drug acarbose (ACA) has been reproducibly shown in that study to increase mouse median lifespan with a larger effect in males than females [2-4]. The largest increase was found when treatment began at 4 months, $22 \%$ in males and $5 \%$ in females [2], but the beneficial effect was still detectable in mice receiving ACA starting at 16 months [3]. The 90th percentile lifespan, a surrogate for maximum lifespan also shows benefits of ACA, with similar magnitudes in both male and female mice [2]. ACA is a competitive inhibitor of $\alpha$-glucosidase and $\alpha$-amylase, resulting in delayed intestinal breakdown of starch when taken with food and reduced postprandial increases in blood glucose. For these reasons, ACA is prescribed for the treatment of type 2 diabetes mellitus [5], and has also been shown to reduce the risk of cardiovascular disease in that population [6].

It is unclear whether the pathways by which ACA extends longevity in mice overlap with those affected by dietary restriction (DR), but several observations have suggested critical differences [2]. Weight loss in ACA mice was more dramatic in females than in males, while the longevity effect is much stronger in males. By contrast, DR effects both weight and lifespan similarly in both sexes. Likewise, the response of fasting hormone FGF21 to ACA treatment was opposite in direction from that induced by DR. In female mice alone, ACA blocked age-related changes in spontaneous physical activity, while DR leads to increases in activity in both sexes. It is therefore justified to suspect that the effects of ACA on longevity are due to pathways distinct from DR.

Besides reducing the absorption of glucose from starch, inhibition of host enzymes by ACA results in increased flow of polysaccharide substrate to the lower digestive system [7], approximately mimicking the effects of fiber consumption. ACA has been shown to raise the concentration of starch in stool $[7,8]$, and the observed increased excretion of hydrogen in breath [9-13] demonstrates that at least some of this substrate is fermented by the gut microbiota. The major byproducts of polysaccharide fermentation in the gut are hydrogen, $\mathrm{CO}_{2}$, and short-chain fatty acids (SCFAs), in particular acetate, butyrate, and propionate. Unsurprisingly, ACA treatment has been observed to increase acetate concentrations in human feces [14] and serum [15], as well as concentrations in portal blood and total amounts in rodent cecal contents [7]. Likewise, in some studies, ACA increased butyrate concentrations in human feces $[8,13,16]$ and serum [15]. ACA also increased propionate concentrations in rat portal blood and total amount in cecal contents [7], as well as total output in feces in humans [14]. These changes were presumably due to changes in the activity and composition of microbial fermenters in the lower gut. Indeed, ACA was found to modulate the composition of the fecal bacterial community in prediabetic humans [17], and both increase the SCFA production potential inferred from metagenomes and lower fecal $\mathrm{pH}$ [18].

Impacts of ACA on microbial fermentation products are of particular interest because SCFAs produced in the gut are known to affect host physiology, with a variety of health effects associated with butyrate and propionate. The role of these microbial fermentation products in host health is reviewed in [19] and [20], and summarized briefly here. Although butyrate and propionate are primarily consumed by the gut epithelium and liver, respectively, they are nonetheless detectable in peripheral blood, and acetate can circulate at substantially higher concentrations [21]. Four G-protein coupled receptors have been shown to respond to SCFAs with varying levels of specificity: FFAR2, FFAR3, HCA2, and OLFR78. All except OLFR78 are expressed in colonic epithelial cells, and each is expressed in a variety of other tissues throughout the body. Similarly, both butyrate and propionate act as histone deacetylase inhibitors which could have broad effects on gene expression through modulation of chromatin structure. In total, these pathways contribute to regulation of cellular proliferation, inflammation, and energy homeostasis, among other processes. The effects of ACA on fermentation products in the gut may, therefore, modulate its overall effects on host physiology.

Despite theoretical expectations and suggestive empirical results in other animal models, no study has looked for direct evidence that some of the longevity enhancing effects of ACA in mice are mediated by the gut microbiota and the SCFAs produced during fermentation. Here we test four predictions of that hypothesis: (1) ACA reproducibly modulates bacterial community composition; (2) the concentrations of SCFAs are increased in ACA-treated mice; (3) community structure is correlated with SCFAs and other metabolites in both control and treated mice; and (4) SCFA concentrations are predictive of lifespan. Fecal samples were analyzed from control and ACA treated mice enrolled in the ITP protocol at three, independent study sites.

\section{Results}

\section{Study population}

Sampled mice are representative of an underlying study population that recapitulates the previously observed sexspecific longevity effects of ACA. Across all three sites, ACA increased the median male survival of the underlying study population by $17 \%$ from 830 to 975 days (log-rank test $P<0.001$, see Fig. 1). Female median survival increased $5 \%$ from 889 to 931 days $(P=0.003)$. 


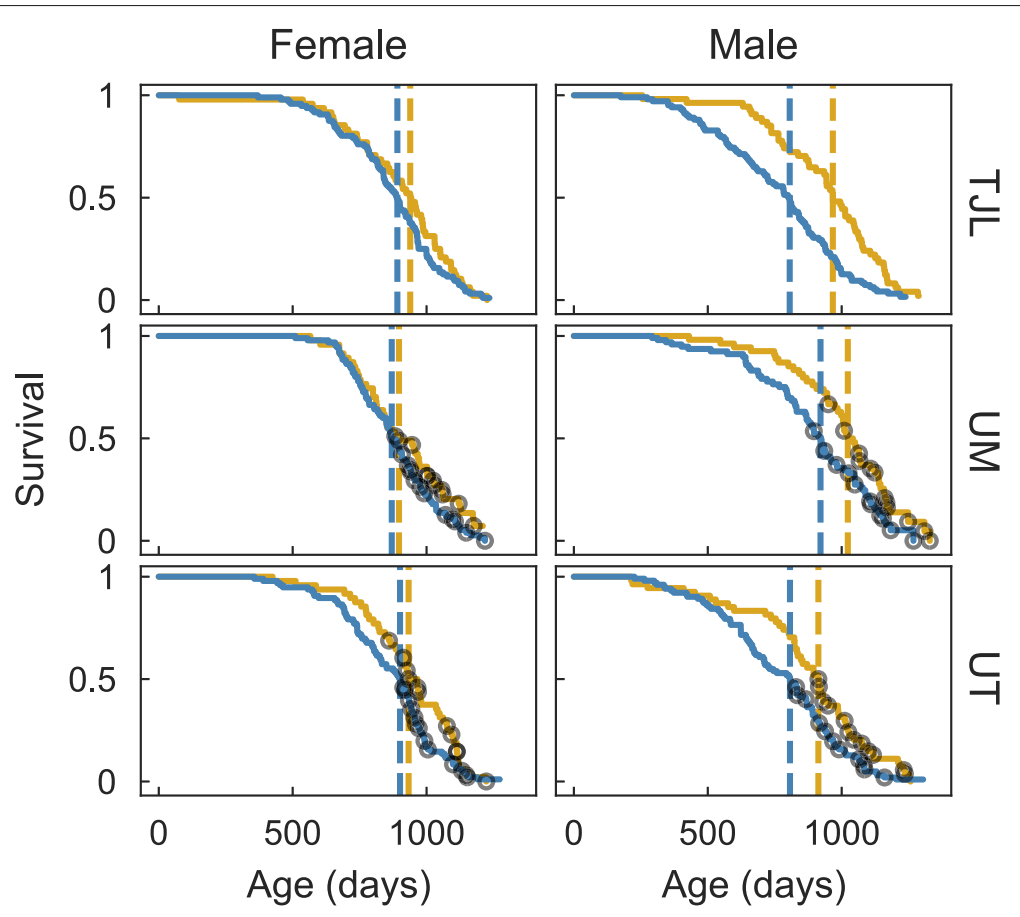

Fig. 1 Survival curves of sampled cohort. Survival curves for mice fed a control diet (blue lines) or mice fed the same diet containing ACA (gold) at each of three sites: TJL, UM, and UT. Median longevity for each group of mice is indicated by a dashed vertical line. Grey circles indicate the age at death for each of the sampled mice at UM and UT. The number of samples summarized by each line is described in Table. 1

These results are consistent with the increased longevity due to ACA previously reported [2]. Fecal samples from 48 mice at each of three study sites-The Jackson Laboratory (TJL), the University of Michigan (UM), and the University of Texas Health Science Center at San Antonio (UT)-were collected between 762 and 973 days of age with a balanced factorial design over sex and treatment group. While having samples collected at the same age for all mice might have reduced some of the observed variability, and this does represent one limitation of the study, the collection window is narrow relative to other biological variation of the mice. For example, the standard deviation in the longevity of mice sampled for this study was 112 days, versus a standard deviation of 50 days for age at sample collection. Visual inspection of the overall survival curves (Fig. 1) confirmed that the longevity of mice sampled for microbiome analyses at UM and UT was representative of the other, surviving, unsampled mice. The total number of mice from which microbiome or longevity data were obtained is summarized in Table 1 . Samples from TJL were not matched to individual mice and longevity measures are therefore not available for the subset described here.

\section{Differences in fecal community in ACA-treated mice}

ACA-treated mice had a substantially different microbial community composition from control mice at all three study sites. In a multivariate analysis of variance on site, sex, and treatment using Bray-Curtis dissimilarities and including all two-way interactions, significant effects were found for treatment (partial $r^{2}=9.6 \%$, PERMANOVA $P<0.001$ ) and site (partial $r^{2}=16.4 \%, P<0.001$ ), as

Table 1 Number of mice sampled

\begin{tabular}{llllll}
\hline Treatment & Site & Sex & Taxonomic & Metabolite & Longevity $^{\mathrm{a}}$ \\
\hline Control & TJL & Female & 12 & 12 & $96^{\mathrm{b}}$ \\
ACA & TJL & Female & 11 & $10^{\mathrm{c}}$ & $48^{\mathrm{b}}$ \\
Control & TJL & Male & 12 & 12 & $102^{\mathrm{b}}$ \\
ACA & TJL & Male & 12 & 12 & $54^{\mathrm{b}}$ \\
Control & UM & Female & 12 & 12 & 96 \\
ACA & UM & Female & 12 & $11^{\mathrm{c}}$ & 48 \\
Control & UM & Male & 12 & 12 & 108 \\
ACA & UM & Male & 12 & 12 & 54 \\
Control & UT & Female & 12 & 12 & 96 \\
ACA & UT & Female & 12 & 12 & 48 \\
Control & UT & Male & 12 & $11^{\mathrm{c}}$ & 102 \\
ACA & UT & Male & 12 & 12 & 54 \\
\hline
\end{tabular}

${ }^{a}$ Microbiome data were not matched to longevity for mice at TJL

${ }^{b}$ Longevity data were compiled for both sampled and unsampled mice in the ITP cohort

'Metabolite data were not compiled for one mouse from each of three groups due to technical irregularities 
well as their interaction (partial $r^{2}=3.4 \%, P<0.001$ ). These statistical results reflect the separation apparent in a principal coordinates ordination (see Fig. 2). A much smaller but still significant effect of sex (partial $r^{2}=1.0 \%$, $P=0.014$ ) was also identified, but there was no interaction between sex and treatment $(P=0.344)$. Despite the unbalanced design, significance of the PERMANOVA was not affected by changing the order of predictors. Based on a test of multivariate homogeneity of variances, dispersion differed between sites (PERMDISP $P<0.001$ ) and sexes $(P=0.023)$, which may bias the PERMANOVA results, but did not differ between treatments $(P=0.425)$. The small effect of sex and the lack of significant sexby-treatment interaction effects suggest that community composition itself, at the level of overall diversity, is not directly responsible for differential effects of ACA on longevity in male and female mice. However, the substantial differences in community composition due to treatment, while not surprising, suggests that the effects of ACA on the microbiome have the potential to modulate host health.

The fecal bacterial community in control mice was dominated by a handful of bacterial families (see Table 2 for details). Across control mice at all three sites, a median of $30 \%$ of sequences were classified as members of the largely uncultured family Muribaculaceae-historically called the S24-7-belonging to the phylum Bacteroidetes [22]. Other abundant families included the Lachnospiraceae (27\%), Ruminococcaceae (14\%), Lactobacillaceae (9\%), and Erysipelotrichaceae (1\%), all of which are classified in the phylum Firmicutes. More than $99.99 \%$ of sequences across all mice were classified at or below the family level. More detailed classification of sequences is provided in Additional file 1.

At a $97 \%$ sequence similarity cutoff, 271 operational taxonomic units (OTUs) had a mean relative abundance across all samples of greater than $0.01 \%$ and an incidence of greater than $5 \%$. Of these, the relative abundance of 113 OTUs differed between treated and control mice, correcting for a false discovery rate (FDR) of $5 \%$. Together, these OTUs account for a median relative abundance of $54 \%$ across both control and treated mice. OTUs differing between sexes or reflecting an interaction between sex and treatment were a substantially less abundant. 5 OTUs were identified after FDR correction that differed significantly in relative abundance between male and female mice, accounting for a median, summed relative abundance of $6 \% .7$ OTUs were found to be subject to an interaction between treatment and sex, with a median relative abundances of $2 \%$. Details of OTUs responsive to treatment, sex, and a treatment-by-sex interaction have been included in Additional file 1.

Differences between control and ACA mice at TJL and UM were dominated by the increased abundance of a single OTU, OTU-1, which had a median relative abundance of $7.7 \%$ in control mice compared to $28.8 \%$ in ACA mice at TJL (Mann-Whitney U test $P<0.001$ ), and $10.4 \%$ compared to $39.0 \%$ at UM $(P<0.001)$ (see Fig. 3). At UT, OTU-1 was higher in ACA-treated mice-a median of $5.4 \%$ and $11.0 \%$ in control and treated mice, respectivelybut this increase was not statistically significant $(P=$ 0.344). Instead, a different OTU, designated OTU-4, was

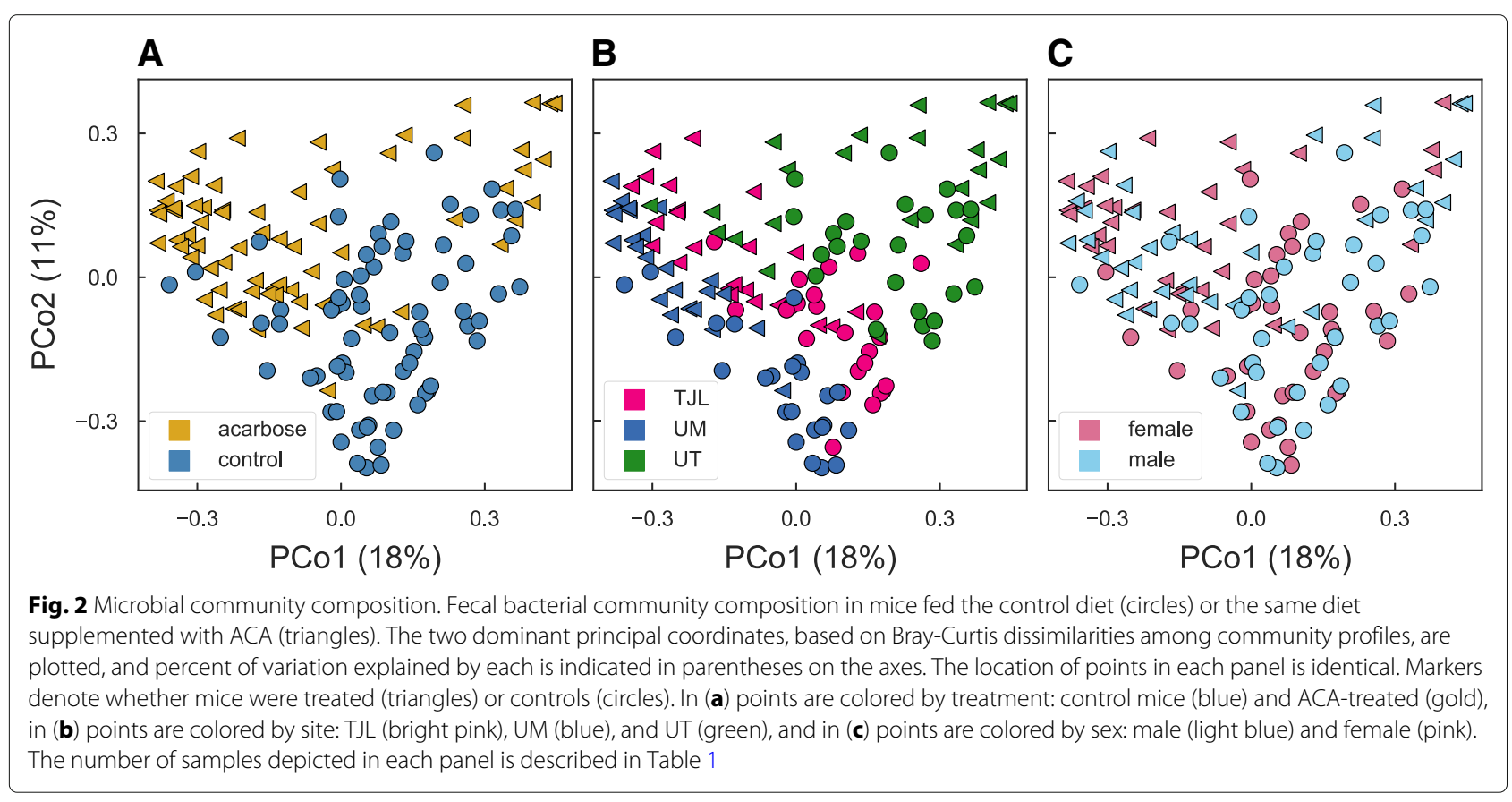


Table 2 Abundance of common bacterial families

\begin{tabular}{|c|c|c|c|}
\hline Family & $\%$ control $^{\mathrm{a}}$ & $\% \mathrm{ACA}^{a}$ & $A C A:$ control $^{b}$ \\
\hline Muribaculaceae & $30.4(21.5,43.3)$ & $48.1^{* *}(35.3,61.8)$ & $1.8^{* *}$ \\
\hline Lachnospiraceae & $26.6(16.3,41.6)$ & $23.9^{\dagger}(9.6,37.4)$ & 1.3 \\
\hline Ruminococcaceae & $14.2(9.0,19.0)$ & $11.6^{*}(6.9,15.6)$ & 1.1 \\
\hline Lactobacillaceae & $9.5(1.2,17.0)$ & $2.6^{*}(1.0,8.2)$ & $0.31^{*}$ \\
\hline Erysipelotrichaceae & $1.4(0.3,6.2)$ & $0.5^{*}(0.2,2.2)$ & $0.42^{\dagger}$ \\
\hline
\end{tabular}

${ }^{a}$ Median and interquartile range of the relative abundance of the top five most abundant bacterial families in control and ACA-treated mice

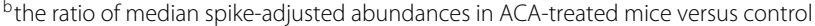
mice

$\doteqdot P<0.1$ via Mann Whitney $U$ test

${ }^{*} P<0.05$

${ }^{* *} P<0.001$

strongly affected by ACA treatment at UT, with a median relative abundance of $6.3 \%$ in control mice that increased to $25.6 \%$ in ACA-treated mice $(P=0.007)$. OTU-4 was nearly absent at TJL and UM, with only one mouse out of 95 having a relative abundance above $0.1 \%$, compared to 39 out of 48 mice at UT. Differences in abundance between sexes were not observed for OTU-1 at TJL or UM, but at UT results were suggestive of an increased abundance of OTU-1 in females $(P=0.076)$ and an increased abundance of OTU-4 in males $(P=0.060)$. Interestingly, their combined abundance did not differ between males and females $(P=0.344)$ at UT. Both OTU1 and OTU-4 were classified as members of the Muribaculaceae, and subsequent phylogenetic analysis confirmed this placement (see Additional file 2). OTU-1 and OTU4 are approximately $90 \%$ identical to each other and to the most closely related cultivar (DSM-28989) over the V4 hypervariable region of the $16 \mathrm{~S}$ rRNA gene. These OTUs are notable both for their high abundance overall, as well as the large difference between control and ACA-treated mice. It is surprising that OTU-4 is common and differentially abundant at UT, while remaining rare at both of the other sites, suggesting that local community composition modulates the effects of ACA. While OTU-1 is made up of multiple unique sequences, the composition within the OTU does not differ substantially with ACA treatment (see Additional file 3).

The increased relative abundance of OTU- 1 and OTU4 in mice treated with ACA appears to be due to greater abundance of these sequences, and is not explained solely by a decrease in other groups. The abundance of taxa in control and treated mice was compared based on the recovery of spiked-in standard relative to the sequence of interest. The median combined spike-adjusted abundance of $16 \mathrm{~S}$ rRNA gene copies from OTU-1 and OTU-4 was 4.3 times greater per gram of feces in ACA-treated mice compared to controls (Mann-Whitney U test $P<$ 0.001 ), suggesting a corresponding increase in population density.

While OTU-1 and OTU-4 are classified to the same family and are similarly affected by ACA, other OTUs in the Muribaculaceae have decreased abundance in treated mice. The combined relative abundance of all other OTUs

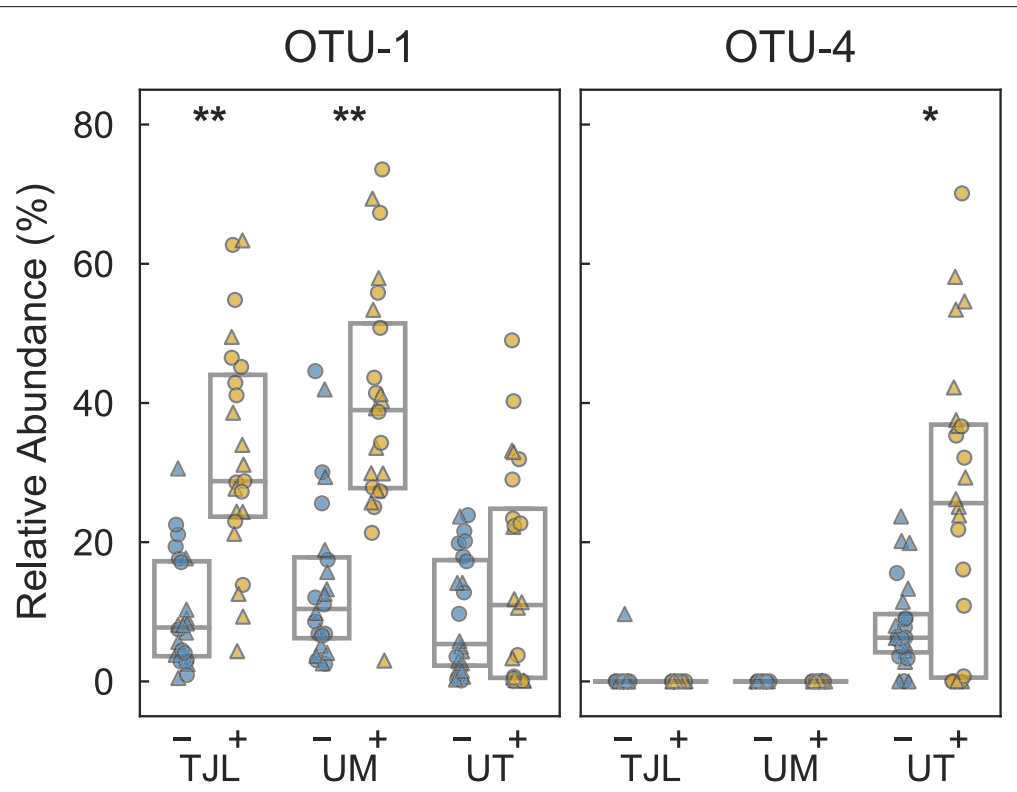

Fig. 3 Abundance of dominant OTUs. Relative abundance of the 16S rRNA gene from two OTUs classified as belonging to the family Muribaculaceae, abundant in ACA-treated mice. Points in each panel correspond with samples collected from individual mice at each of three replicate study sites. Samples were obtained from mice fed either the control diet (blue, labeled '-') or the same diet supplemented with ACA (gold, labeled ' + '). Markers indicate the sex of the mouse: male (triangle) or female (circle). The number of samples illustrated in each panel is described in Table. 1. Boxes span the interquartile range and the internal line indicates the median. ${ }^{*}: P<0.05$, ${ }^{*}$ : $P<0.001$ by $M a n n-W h i t n e y ~ U$ test) 
in the family-excluding OTU-1 and OTU-4-was 8.3\% in treated mice versus $16.8 \%$ of sequences in control mice (Mann-Whitney U test $P<0.001$ ). The median combined spike-adjusted abundance of all other Muribaculaceae OTUs was 0.5 times the median in control mice $(P=$ 0.001 ), suggesting a decrease in the population density of these taxa. This is consistent with competition between OTUs in this family.

Three of the five most abundant families all exhibit decreased relative abundance in ACA treated mice (see Table 2). However, the large increase in abundance of OTU-1 and OTU-4 suggests that some changes in the relative abundance of other taxa may be the result of compositional effects, rather than decreased density. For instance, although the relative abundance of Ruminococcaceae was lower in ACA-treated mice, the spike-adjusted abundance was little changed $(P=0.327)$, emphasizing the value of this complementary analysis. Conversely, decreased relative abundance was matched by decreased spike-adjusted abundance for both the Lactobacillaceae $(P=0.014)$ and the Erysipelotrichaceae $(P=0.063)$.

ACA-treated mice exhibited decreased fecal community diversity. The median Chao1 richness estimate was decreased from 229 in control mice to 199 in treated mice (Mann-Whitney U test $P<0.001$ ). The Simpson's evenness index was also lower in ACA mice: 0.044 versus 0.075 in controls $(P<0.001)$. This reduced richness and evenness is not surprising given the much greater abundance of a single OTU in treated mice at each site. To understand changes in diversity while controlling for compositional effects, we measured the effect of ACA ignoring counts for OTU-1 and OTU-4. While Simpson's evenness was not decreased by treatment in this fraction of the community $(P=0.26)$, the Chao1 richness-subsampling to equal counts after partitioning-was decreased $(P=$ 0.005), suggesting that the bloom of OTU-1 and OTU-4 may have, in fact, resulted in the local extinction of rare community members.

\section{Changes in fecal metabolite concentrations}

The effects of long-term ACA treatment on fecal metabolite profiles was variable across study sites, but with increased concentrations of propionate at all three (see Fig. 4). Consistent with the expected increase in starch in the lower gut, glucose concentrations were higher in ACA-treated mice at UM (from a median of $6.1 \mu \mathrm{mols} / \mathrm{g}$ in control mice to 12.9 in treated mice, Mann-Whitney U test $P<0.001$ ), and UT (2.8 to $9.1, P<0.001$ ). At TJL, while the statistical test did not surpass the significance threshold, there was also an observed increase in glucose concentrations (6.6 to 9.1, $P=0.069$ ). However, this presumed increase in fermentable substrate did not result in increased total SCFA-here defined as the sum of acetate, butyrate, and propionate concentrations-at either TJL (22.4 to $18.8, P=0.443$ ), or UM (19.1 to 24.8 , $P=0.092$ ). Interestingly, total SCFA was sharply higher in ACA treated mice at UT alone (17.7 to 31.9, $P<0.001$ ). This same pattern of variation across sites was found in the effects of ACA on fecal acetate and butyrate concentrations, where statistically significant increases were only found at UT (acetate: $P=0.003$, butyrate: $P<0.001$ ), but not at either of the other two sites. Fecal propionate concentrations, on the other hand, were elevated in ACA mice at all three study sites, from a median of 0.93 to 1.9 at TJL $(P=0.001)$, from 1.1 to 2.5 at $\mathrm{UM}(P<0.001)$, and from 1.9 to 2.9 at UT $(P=0.008)$.

The consistent increase in fecal propionate was matched by consistently lower concentrations of one of its metabolic precursors, succinate, at all three sites, from 2.8 in control to 1.5 in ACA-treated mice at TJL ( $P=$ $0.009)$, from 3.0 to 1.2 at UM $(P<0.001)$, and from 3.1 to 2.1 at UT $(P=0.022)$. Median lactate concentrations were also lower in ACA treated mice, although this was only statistically significant at UT $(P=0.002)$. It is surprising that these fermentation intermediates are reduced, given the expected increase in available polysaccharide. It is possible that their concentrations reflect greater consumption in downstream pathways, or perhaps ACA directly inhibits the metabolism and growth of relevant bacteria; such effects have been previously reported for in vitro fermentations of starch with human fecal slurries [8]. Concentrations of formate, valerate, isobutyrate, and isovalerate were generally below the detection limit. Fresh pellet weight was increased from a median of 36 to $74 \mathrm{mg}(P<0.001)$.

Given the inconsistant observation of changes in metabolite concentrations with ACA across study sites, we tested for treatment-by-site interaction effect using an ANOVA framework, with log-transformed metabolite concentrations to better accommodate distributional assumptions. Using this approach, significant interactions were found for glucose $(P=0.005)$, and acetate $(P=$ $0.044)$, and results were suggestive for succinate $(P=$ $0.069)$, butyrate $(P=0.071)$, and total SCFA $(P=0.053)$. Treatment-by-site interaction effects were not found for lactate $(P=0.266)$ or propionate $(P=0.542)$.

Differences in SCFAs between sexes are particularly interesting given the greater longevity effects of ACA in male mice. In a log-linear model that included terms for site, sex, and treatment, as well as all other two-way interactions, including a treatment-by-sex interaction significantly improved the fit of the model for propionate (LRT $P=0.020$ ), but not for butyrate or acetate. This interaction results in a larger difference in propionate concentrations for male mice (from an overall median of 1.4 $\mu \mathrm{mols} / \mathrm{g}$ in control mice to 2.7 in ACA) than for female mice (from 1.0 to 1.9 ) with ACA treatment. The significance of the interaction term was not corrected for 


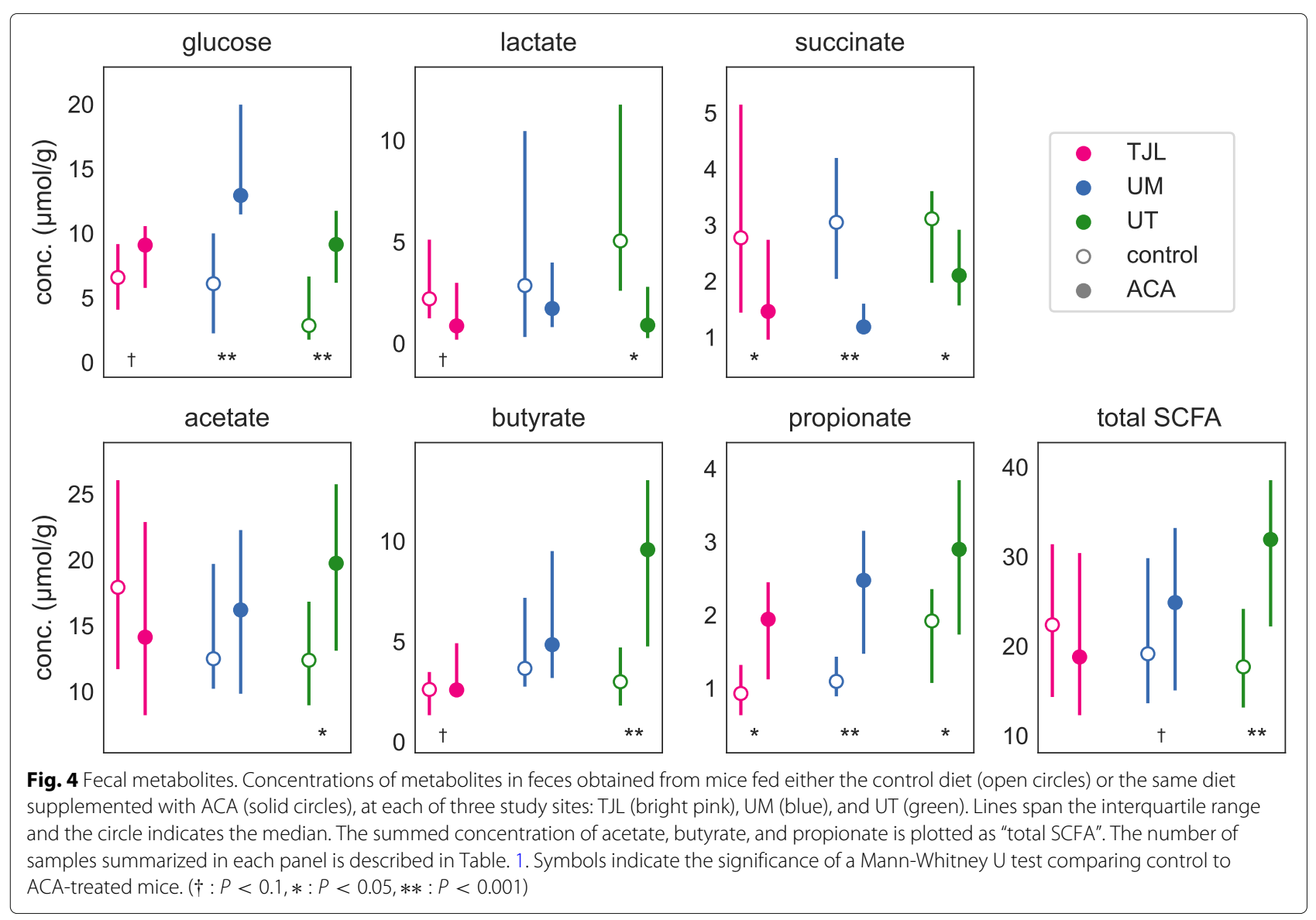

multiple testing, and therefore additional studies would greatly increase our confidence in this result.

\section{Community predictors of fecal SCFA concentrations}

As both community composition and the effects of ACA on metabolite concentrations in feces varied greatly between study sites, we explored the relationship between microbial abundance and metabolite concentration. Community composition was correlated with metabolite concentrations in both control and ACA-treated mice. Numerous strong correlations were detected between the spike-adjusted abundance of $16 \mathrm{~S}$ rRNA copies from the most common bacterial families and the concentrations of SCFAs and lactate. Notably, Muribaculaceae abundance was particularly strongly correlated with propionate concentrations in both control (Spearman's $\rho=0.36, P=$ 0.002; see Fig. 5) and ACA mice $(\rho=0.64, P<0.001)$. Likewise, Lachnospiraceae were correlated with butyrate ( $\rho=0.61$ in control and 0.77 in ACA, $P<0.001$ for both), and Lactobacillaceae with lactate concentrations $(\rho=0.63$ in control and 0.67 in ACA, $P<0.001$ for both). Strikingly, concentrations of acetate and butyrate were especially correlated with each other $(\rho=0.67$ in control and 0.80 in ACA, $P<0.001$ for both). What's more, all of these correlations were found in treated mice even when independently tested at each of the three sites, suggesting that they cannot be explained by simple associations between metabolite concentrations and community composition at the site level.

Although our study was not an unambiguous test, these results support the hypothesis that the fecal metabolite response to treatment is dependent on the population density of relevant microbes in the gut community. Similarly, environmental and host factors that promote or inhibit the growth of particular community members would be expected to modulate the response.

To identify key players in these associations, we examined the relationship between metabolite concentrations and the spike-adjusted abundances of OTUs. Based on a least absolute shrinkage and selection operator (LASSO) regression analysis, the abundances of a number of OTUs can be used to predict concentrations of propionate, butyrate, acetate, and lactate even after accounting for treatment, sex, and study site. Consistent with the correlations found between Muribaculaceae abundance and propionate, OTU-1 and OTU-4 were identified as predictors 
A

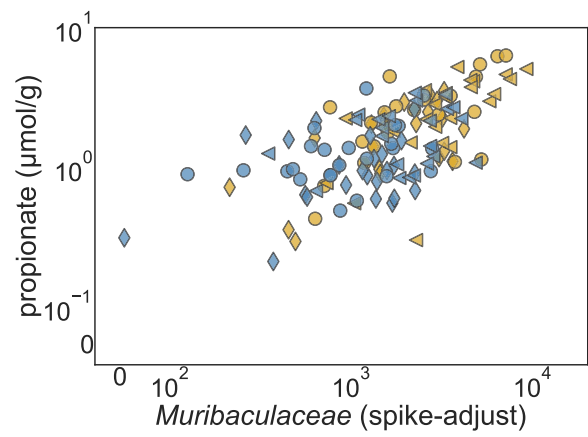

C

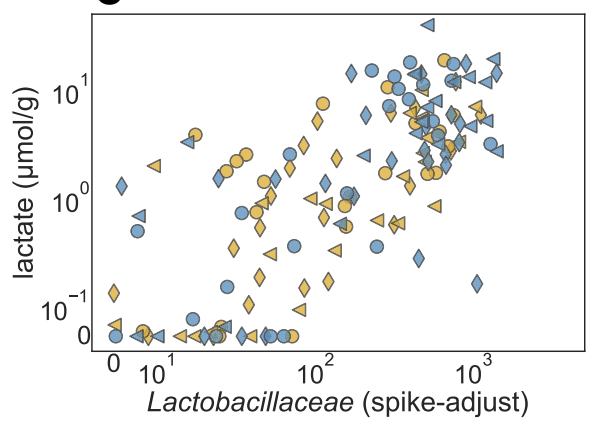

B

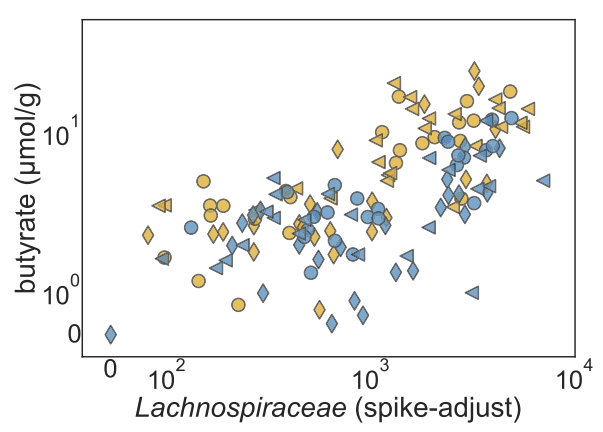

D

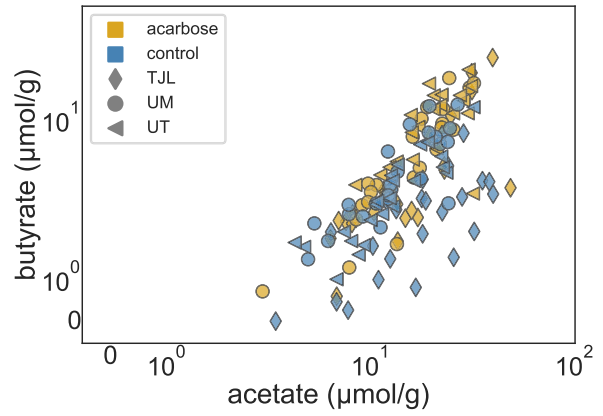

Fig. 5 Microbiota/metabolite correlations. Correlations among metabolite concentrations in feces and family level, spike-adjusted 16S rRNA gene abundances. Points correspond with samples collected from individual mice and colors indicate whether they were obtained from mice fed the control diet (blue) or the same diet supplemented with ACA (gold). Markers indicate the site where the mouse was housed: TJL (diamond), UM (circle), or UT (triangle). Metabolite concentrations are reported normalized to feces wet weight, and abundances are in spike-equivalent units. Values are on a linear scale between 0 and the subsequent tick label, above which, points are plotted logarithmically. The number of samples illustrated in each panel is described in Table 1

of increased propionate, along with a third taxon, OTU5 , also classified as a member of the family. For both butyrate and acetate, OTUs classified as members of both the Lachnospiraceae and Ruminococcaceae were most predictive of increased concentrations. Unsurprisingly, the most abundant OTU classified as a member of the Lactobacillaceae, OTU-2, was found to be highly predictive of increased lactate. However, 8 OTUs were also associated with decreased lactate concentrations, most of which were among those associated with increased butyrate and acetate. Among other explanations, this is consistent with these taxa either being inhibited by lactate or being lactate utilizers, which are likely to be producing SCFAs as secondary fermentation products. Regression coefficients for each metabolite are included in Additional file 1. Overall, results were both consistent with a priori expectations, and useful for generating hypotheses about which taxa might be associated with the generation of fermentation products.

\section{Fecal SCFA concentrations as predictors of longevity} Given the documented health benefits of SCFAs in the gut (reviewed in [19]) and their increased levels in ACA- treated mice, we tested the relationship between the acetate, butyrate, and propionate concentrations in feces, and the lifespan of individual mice. Lifespans of fecal donors were not available for mice at TJL, so survival analyses were carried out only with UM and UT mice, and effect sizes are reported for SCFAs as standardized hazard ratios (HRs). Due to the reduced number of mice sampled for this study, data were pooled across sexes and sites. The shared effects of the design parameterstreatment, sex, and study site-on both SCFAs and longevity, were accounted for by including terms for these covariates as well as their two and three-way interactions. Analyses reinforcing our interpretations are discussed in Additional file 4. Tested individually against this null model, an association between longevity and propionate was found (standardized HR of $0.727, P=0.031$ ), but no relationship was observed with butyrate $(P=0.240)$ or acetate $(P=0.742)$. However, when the model was fit with all three SCFAs simultaneously, each was found to be associated with longevity $(P=0.012,0.030,0.042$ for propionate, butyrate, and acetate, respectively). Coefficients for SCFA covariates in this full model suggest a positive association with longevity for both propionate and 
butyrate (standardized HR of 0.674 and 0.586 , respectively). Interestingly, a negative association was found with acetate (standardized HR of 1.576) using this model. The discrepancy between this result and the lack of association when butyrate and acetate are each tested alone likely reflects the strong positive correlation between acetate and butyrate concentrations, masking their individual, opposing associations with longevity. The overall fit of the full model was improved compared to the null model with only design covariates (likelihood ratio test, $P=0.023$ ).

\section{Discussion}

ACA, by inhibiting the enzymes responsible for starch degradation in the small intestine, is expected to increase the availability of this polysaccharide to the microbiome. The resulting increase in SCFA production may contribute to the effects of ACA on health. Despite previous observations in humans and rats that ACA results in substantial changes to the community structure $[8,17]$ and fermentation products $[7,8,13,16]$ of the gut microbiota, a link between these effects on the microbiome and longevity has not been established. Here we present one of the first studies to combine bacterial community surveys with measurement of fecal metabolites in ACA treated mice (with the notable exception of [23]), as well as the first to pair these data with lifespan, allowing us to explore the role of the microbiome in increased longevity. By considering mice at three parallel study sites, we are also able to account for variation in the composition and response of the gut microbiota and its impact on study reproducibility.

Our results support all four predictions that we set out to test: ACA was found to affect both (1) the composition of bacterial communities and (2) SCFAs in mouse feces, (3) the abundances of individual taxonomic groups were correlated with concentrations of fermentation products, and (4) the concentrations of fecal SCFAs were associated with variation in mouse longevity.

While it is unsurprising that an increased flux of starch to the large intestine affected the gut microbiota and their fermentation products, the specific impacts could not be easily predicted, especially since ACA can also inhibit bacterial enzymes, and this can be variable across taxa [24]. The increased relative abundance in ACA-treated mice of the dominant OTU-OTU- 1 at UM and TJL and OTU4 at UT-was dramatic: one or the other was increased approximately 4-fold at all three sites and in multiple samples more than half of sequences belonged to these OTUs. A cursory BLAST search reveals that sequences identical to OTU-1 have been previously recovered in published studies (e.g. [25] and [26]); in [27] the sequence was found at high relative abundance in the brains of mice that had undergone sepsis. It was notable that OTU-1 did not respond to ACA at UT, while its increased abundance was so striking at UM and TJL. Our results appear to constrain the potential explanations for this observation. OTU-1 was present and abundant at all three sites; The abundance in control mice was lowest at UT, although the median there was still greater than $5 \%$. While it is not possible with the data presented here to rule out genomic differences of OTU-1 among sites, a similar composition of unique 16S rRNA gene sequences made up this cluster at all three (see Additional file 3). On the other hand, OTU-4 was at very low abundance, with no reads in a majority of samples, at UM and TJL where OTU-1 did respond to ACA. These results suggest that both OTUs respond to ACA in the same way, with OTU-4, when it is sufficiently abundant, inhibiting the response of OTU1 , potentially through resource competition. Both OTUs are in the same family, the Muribaculaceae, but are not the same species or genus by the traditional similarity thresholds, sharing only $90 \%$ identity over the sequenced fragment. The differential response of these OTUs among sites illustrates the importance of each site's local "metacommunity" in determining the microbial community's response to environmental perturbations.

Pronounced differences in the resident microbial communities of different hosts may contribute to challenges in translating results from mice and other model organisms to humans. A comparison of bacterial community composition in feces in prediabetic people before and during a 4-week ACA treatment period did not reveal changes of the magnitude reported here [17], although this may reflect the limited duration of treatment. Interestingly, in that study Lactobacillaceae abundance increased with ACA, while we observed this family to be depleted in treated mice. The abundance of the Muribaculaceae was not reported. Although this family is common in mice and have been previously shown to respond to diet, the clade is substantially less abundant in most human samples [28]. However, the prevalence of Muribaculaceae may be under-reported in the literature, as the Ribosomal Database Project [29] does not include the family and classification using this database assigns sequences to the Porphyromonadaceae instead [30,31]. Historically, two other names have also been used for this clade: the "S24-7" (from an early environmental clone [32]), and "Candidatus Homeothermaceae" (proposed in [28]). While the initial isolation of Muribaculum intestinale YL27 [22], and subsequently five additional strains [31], has begun to explore this largely uncharacterized clade, additional cultivars will be vital for understanding the function and ecology of the family. Nonetheless, genomes assembled from metagenomes suggest that populations of Muribaculaceae are equipped with fermentation pathways to produce succinate, acetate, and propionate [28], and that the family is composed of metabolic guilds, each specializing on the degradation of particular types of polysaccharides: 
plant glycans, host glycans, and $\alpha$-glucans [28, 31]. This suggests that the Muribaculaceae may occupy a similar set of niches in mice as do Bacteroides species in humans. The Muribaculaceae and Bacteroides are both in the order Bacteroidales. Bacteroides also specialize in the fermentation of polysaccharides [33], and at least some of the most common species in the human gut are known to produce succinate, acetate, and propionate from the fermentation of polysaccharides [19, 34-36]. Unlike the patterns observed here for Muribaculaceae in mice, the abundance of Bacteroides decreased with ACA treatment in one study in humans [17], suggesting that the microbially mediated effects of ACA may fundamentally differ between these hosts.

Besides hypotheses based on genome content, the correlation between total Muribaculaceae abundance and propionate concentrations and the specific association with OTU- 1 and OTU-4 found in the LASSO analysis suggest that both OTUs, and perhaps other Muribaculaceae species in this study, ferment starch to propionate. This also supports the hypothesis, discussed above, that both OTUs occupy overlapping niches. Although increased butyrate concentrations have been frequently reported with ACA treatment $[7,8,13,15,16,23]$, elevated concentrations of propionate have been observed in just one previous study using portal blood in rats [7]. Studies in humans have instead found decreased or no change in fecal $[8,13,14]$ or serum [15] propionate concentrations with ACA. Decreased propionate has been attributed to preferential production of butyrate from starch fermentation [37, 38] or inhibition of propiogenic bacteria by ACA [8]. Our observation of increased propionate was robust and reproduced at all three sites. Interestingly, in the only other study to pair SCFA measurements with taxonomic survey results, which also failed to find a significant increase in propionate, the relative abundance of the Muribaculaceae was decreased as well [23]. If these conflicting findings reflects both the greater initial abundance and enrichment in our study of the Muribaculaceaeespecially OTU-1 and OTU-4-it demonstrates the value of measuring both community composition and metabolite concentrations in the same samples.

Intriguingly, the Muribaculaceae have also been observed to be enriched in mice receiving fecal transplants from donor mice on calorie restricted diets, and metabolic improvements were also observed in recipients [39]. Likewise, the response of the in situ bacterial community suggests a possible role of the microbiome in the increased longevity of calorie restriced mice [40].

SCFAs are commonly suggested to act as intermediaries between the gut microbiota and host physiology [19]. While our study was not designed to provide a causal test of effects of SCFAs on longevity, and the power of our analysis was limited, a statistical association between
SCFA concentrations and mouse lifespan supports an interpretation that is consistent with an extensive literature on the health benefits of butyrate and propionate [19]. The positive associations with propionate could, for instance, be due to FFAR2 dependent regulation of inflammation [41, 42]. Likewise, both butyrate and propionate activate FFAR3-which can result in increased secretion of PYY and GLP-1 [43, 44] with implications for glucose homeostasis [45] - and inhibit histone deacetylases [46], with downstream effects on both inflammation [47] and carcinogenesis [48]. Interestingly, treatment with the polysaccharide inulin, which increases fecal SCFA concentrations in mice [49], was also found in one study to increase survival in rats [50]. That SCFA concentrations were associated with longevity above and beyond the effects of ACA, study site, and sex, further supports a role of the microbiota and their metabolites in the longevity enhancing effects of ACA treatment.

It is somewhat surprising, however, that a single fecal sample taken, in some cases, several months before death, could be predictive of longevity. The association reported here could reflect other, unmeasured, changes in the gut microbiome or host physiology. Concentrations of metabolites in feces are an integration of both production and consumption rates along the length of the lower gut, and may not reflect host exposure nor the strength of host physiological response. It is also important to note that, since all mice in this study at the time of sampling were of an age close to the median lifespan of control individuals, the results are only relevant to mechanisms of aging in late-life and should not be extrapolated to young mice. While suggestive, the results presented here are unable to rule out a number of alternative mechanisms which may confound our analysis of the relationship between SCFA concentrations and survival. For instance, variation in starch digestion between mice could also produce the observed correlations between host physiology (through blood glucose regulation) and fecal metabolites, without any direct causal relationship between the two. Similarly, changes in the measured fermentation products may reflect effects of ACA on the concentrations of other, unmeasured microbial metabolites; luminal polyamine concentrations, for instance, have been linked to longevity in mice [51]. Experimental tests of a causal role for microbial products, such as SCFAs, in longevity are challenging, as they require controlled manipulation of intestinal concentrations for the lifetime of a mouse.

It is notable that both community composition and SCFA concentrations were distinct at each of the three replicate study sites. Given the robust correlations between bacterial abundance and metabolites, as well as treatment-by-site interactions in SCFA concentrations, attempting to generalize results from a single study site would be misguided. Our results suggest that such 
variation can contribute to the challenges in reproducing and translating results from animal models even in studies that do not explicitly consider the microbiome [52-55]. This reinforces the importance of replication and multisite studies, and illustrates the value of such designs for testing the role of the microbiome in outcomes.

Due to the preferential enhancement of longevity by ACA in male mice we sought to identify aspects of the gut microbiome that responded differently in male and female mice (i.e. interaction effects), as these might suggest mechanistic explanations for differences in longevity effects [2]. While we do not believe that the magnitude of sex-by-treatment interactions observed for various aspects of the microbiome were sufficiently pronounced to fully explain the differential effect of ACA on lifespan, our search was limited by sample size, variability between study sites, and the large number of features being tested. Nonetheless, ACA was found to increase propionate concentrations more in male mice than females, a statistically significant pattern before correction for multiple testing, and the relative abundance of a handful of OTUs seem to have also been subject to an interaction.

Mechanisms unrelated to the gut microbiome have been proposed for the effects of ACA on lifespan. Because ACA reduces the postprandial glucose spike observed in mice and humans, hypotheses emphasizing the reduction of harmful effects associated with these transient surges have been most commonly invoked. Studies of UM-HET3 mice given ACA from 4 to 9 months of age suggested that mean daily blood glucose levels are minimally affected, but that absorption of glucose was both slower and longer lasting [2]. Interestingly, fasting insulin level in ACA-treated males are much lower than those in control males, consistent with an improvement in insulin sensitivity [2]. This reduction was not seen in females, where insulin levels in controls were lower than in control males and similar to those in ACA-treated males [2,56], presenting one possible explanation for the stronger longevity benefit in males. Still, the connection between this modulation of postprandial glucose-with or without improved insulin sensitivity-and extended longevity is far from certain.

The work presented here explores a different hypothesis: that health benefits in ACA mice are related to changes in the activity of microbial communities in the gut associated with the increased influx of starch, and possibly attributable to known health effects of microbial metabolites, including SCFAs. Observed changes in both community composition and fermentation products due to ACA treatment, along with the statistical association between fecal SCFA concentrations and longevity, are consistent with this hypothesis, and provide a steppingstone for future studies. Interestingly, SCFAs themselves have well documented effects on glucose homeostasis (reviewed in [57] and [58]). The two explanations are therefore not mutually exclusive, and the effects of ACA on longevity may be mediated by both glucose physiology and microbial activity in the gut.

\section{Conclusions}

Here we have tested four predictions of a proposed model connecting ACA to lifespan via the gut microbiome. We demonstrate that ACA reproducibly modulates the composition of the microbiota, as well as the concentrations of fermentation products, increasing the abundance of propionate in particular. In addition, we provide evidence that the structure of the microbial community is an important factor in the composition of metabolites produced. Finally, we observe an association between SCFA concentrations in feces and survival, suggesting a role of the microbiome in the life-extending properties of ACA. Together, these results encourage a new focus on managing the gut microbiota for host health and longevity.

\section{Methods}

\section{Mouse housing and ACA treatment}

Mice were bred and housed, and lifespan was assessed as described in [2]. Briefly, at each of the three study sites, genetically heterogeneous, UM-HET3, mice were bred by a four-way cross as previously described in [59], from CByB6F1/J females and C3D2F1/J males purchased annually from the Jackson Laboratories. After weaning, mice were fed LabDiet (TestDiet Inc.) 5LG6 produced in common batches for all sites. At 42 days of age, electronic ID chips were surgically implanted, under isoflurane inhalation anesthesia, and treatment randomly assigned to each cage housing four mice to a cage for females and three to a cage for males. ACA-treated mice were fed the same chow amended with 1000 ppm ACA (Spectrum Chemical Manufacturing Corporation) from 8 months of age onwards. Mice were transferred every 14 days to fresh, ventilated cages with water provided in bottles. Colonies at all three sites were assessed for infectious agents four times each year, and all tests were negative for the entire duration of the study. For humane reasons, mice believed to be within $48 \mathrm{~h}$ of death were euthanized by $\mathrm{CO}_{2}$ asphyxiation, and all mice died in this way or from natural causes by the end of the study.

\section{Sample collection and processing}

Fresh fecal pellets were collected directly from mice between 762 and 973 days of age and frozen at $-80^{\circ} \mathrm{C}$. We did not control the time of day at collection. While differences in age and collection time could have added variability to SCFA concentrations, both were similarly distributed for the different treatment groups, so they are unlikely to confounded our analyses. To eliminate potential cage effects from co-housed mice [54], samples 
were obtained from no more than one randomly selected mouse per cage. A total of 144 samples were collected from 12 male and 12 female mice in both control and ACA treatment groups at each of the three sites. Samples were shipped on dry ice and then stored, frozen, until processing. For approximately the first half of samples, we extracted the soluble fraction by homogenizing pellets with $200 \mu \mathrm{L}$ of nuclease-free water. For the remaining samples, we instead used a 1:10 ratio (weight:volume), with a maximum volume of $1.5 \mathrm{ml}$. This was found to improve quantification in higher weight samples. While SCFA concentration estimates were higher when using the amended protocol, the order of sample extraction was fully randomized, so it is unlikely to have biased our interpretations. Homogenized samples were centrifuged at $10,000 \times \mathrm{g}$ for 10 minutes to separate soluble and solid fractions. The supernatant was then serially vacuum filtered, ultimately through a $0.22 \mu \mathrm{m}$ filter, before HPLC analysis. The solid fraction was frozen prior to DNA extraction. Four samples were excluded from chemical analysis and one from DNA analysis due to technical irregularities during sample processing.

Prior to DNA extraction, fecal pellet solids were thawed and, where necessary, subsampled for separate analysis. To move beyond relative abundance, solids were weighed and spiked with $10 \mu \mathrm{L}$ aliquots of prepared Sphingopyxis alaskensis strain RB2256-an organism not found in mouse feces-in order to compare 16S rRNA gene abundance between samples $[60,61]$. The spike was prepared as follows: a 1:200 dilution of a stationary phase $S$. alaskensis culture was grown at room temperature for approximately 44 hours in R2B medium with shaking. This culture was harvested at a final OD420 of 0.72 before being rinsed in PBS and resuspended-5-fold more concentrated-in $20 \%$ glycerol in PBS (v/v). Aliquots of these cells were stored at $-20^{\circ} \mathrm{C}$ before extraction and sequencing. Spiked fecal samples were homogenized in nuclease free water at a ratio of 1:10 (w/v). DNA was extracted from $150 \mu \mathrm{L}$ of this mixture using the MoBio PowerMag Microbiome kit.

\section{Chemical analysis}

The chemical composition of samples was assessed on a Shimadzu HPLC (Shimadzu Scientific Instruments) equipped with an RID-10A refractive index detector. $30 \mu \mathrm{L}$ injections were run on an Aminex HPX-87H column (Bio-Rad Laboratories, Hercules, CA) at $50^{\circ} \mathrm{C}$ with $0.01 \mathrm{~N} \mathrm{H}_{2} \mathrm{SO}_{4}$ mobile phase and a flow rate of $0.6 \mathrm{ml} /$ minute. External standards were run approximately daily containing acetate, butyrate, formate, glucose, lactate, propionate, and succinate at 8 concentrations between $0.1 \mathrm{mM}$ to $20 \mathrm{mM}$. Due to the complexity of the chromatogram, the identity and area of retained peaks was curated manually, assisted by the LC Solutions Software
(Shimadzu Scientific Instruments) Standard curves were fit using weighted regression (inverse square of the concentration), and, for all compounds except propionate, without an intercept.

\section{S rRNA gene sequencing and analysis}

The V4 hypervariable region of the 16S rRNA gene was amplified from extracted DNA (as described in [62]), and sequenced on an Illumina MiSeq platform using a MiSeq Reagent Kit V2 500 cycles (cat\# MS-102-2003). Amplicon sequences were processed with MOTHUR (version 1.39.4 [63]) using a protocol based on the $16 \mathrm{~S}$ standard operating procedures [62]. Scripts to reproduce our analysis can be found at [64]. After fusing paired reads, quality trimming, and alignment to the SILVA reference database (Release 132 downloaded from [65]), the vast majority of $16 \mathrm{~S}$ rRNA gene sequences were between 244 and $246 \mathrm{bp}$. Sequences were classified using the method of Wang et al. [66] as implemented in MOTHUR and with the SILVA non-redundant database as a reference [67]. We clustered sequences into OTUs using the OptiClust method [68] at a 97\% similarity threshold. We counted and removed sequences classified as $S$. alaskensis, the spikedin standard, before further analysis. We did not attempt to assess the exact number of $16 \mathrm{~S}$ rRNA gene copies spiked into samples. Instead, spike-adjusted abundance was defined in units based on the standardized spike ( $\mu \mathrm{L}$ spike equivalents/g sample) and estimated using the formula:

RelativeAbundance $\times \frac{\text { (TotalEndemicReadCount } \times \text { SpikeVolume })}{(\text { TotalSpikeReadCount } \times \text { SampleWeight })}$

Family level abundance was calculated as the summed abundance of all sequences clustered in OTUs classified to that family. OTU counts were randomly subsampled to the minimum number of reads before calculating $\alpha$ diversity metrics, but were not subsampled for other analyses, as these are robust to differences in sequencing depth. A single, independent realization of random subsampling was used for each richness calculation. A search of the NCBI non-redundant nucleotide database for related sequences from cultured bacteria was carried out using the BLASTn web tool [69] searching the nonredundant nucleotide database with default parameters and excluding sequences from uncultured organisms.

\section{Statistical analysis}

A $0.05 \mathrm{p}$-value threshold was used to define statistical significance, with values below 0.1 considered "suggestive". Except where specified, p-values are not corrected for multiple testing. Due to the risk of violating distributional assumptions, univariate comparisons between groups were done using the non-parametric Mann-Whitney $\mathrm{U}$ 
test. Differences in multivariate community composition and dispersion were tested using PERMANOVA (adonis) and PERMDISP (betadisper) respectively, both implemented in the vegan package (version 2.4-6 [70]) for the $\mathrm{R}$ programming language. Bray-Curtis dissimilarity was used as the $\beta$-diversity index.

Differences in the relative abundance of individual OTUs were surveyed using the DESeq2 package (version 1.18.1 [71]) for R, and fitting a model that included terms for treatment, sex, site, and the interaction between treatment and sex. So as to keep valuable distributional information, all OTUs found in at least two samples were included in the initial analysis, with p-values calculated using a Wald test. However, FDR correction using the Benjamini-Hochberg procedure [72] excluded "rare" OTUs-those with mean relative abundance less than $0.01 \%$ or detected in fewer than $5 \%$ of samples-in order to maintain statistical power by independently reducing the number of tests.

Interactions between sex and treatment in fecal SCFAs were assessed for log-transformed concentrations. The small number of zeros were replaced with half the lowest detected concentration for that metabolite. Interactions were tested in an ANOVA that also included terms for site, sex, and treatment. LASSO regressions of the three SCFAs and lactate against spike-adjusted OTU abundances were performed using the scikit-learn library for Python (version 0.18.2 [73]) and log-transformed concentrations after adjustment for site, sex, and treatment. OTUs detected in more than $5 \%$ of samples and with mean abundance greater than $0.01 \%$ were included. The LASSO parameter was determined by randomized 10-fold cross-validation, optimizing for out-of-bag $\mathrm{R}^{2}$. For each metabolite we confirmed that OTU abundance information improved predictions by testing the Spearman's rank correlation between true values and out-of-bag predictions of the best model using a Student's t-distribution approximation [74] and a $P=0.05$ significance threshold. While this type of regularized regression is primarily useful for constructing predictive models, and biological interpretation can be challenging, non-zero regression coefficients are suggestive of covariates that are among the most strongly associated with a response. Proportional hazards regression was carried out using of the survival package (version 2.41-3 [75]) for $\mathrm{R}$, and the day of fecal sampling as the entry time. All sampled mice were dead at the time of analysis and right-censoring was therefore not used. Standardized HRs reported for SCFAs are based on concentrations that have been centered around 0 and scaled to a standard deviation of 1 .

Various experimental data and metadata, including mouse ages at sampling, pellet weights, and raw HPLC peak data, are all freely available on GitHub [64], along with code necessary to reproduce our analyses.

\section{Additional files}

Additional file 1: Summary statistics, associations with design parameters and metabolites, and inferred taxonomic identity of common bacterial OTUs. Table includes all OTUs with mean relative abundance greater than $0.01 \%$ and found in greater than $5 \%$ of samples. (XLSX $51 \mathrm{~kb}$ )

Additional file 2: Taxonomic analysis of two dominant OTUs. (PDF $58 \mathrm{~kb}$ ) Additional file 3: Relative abundance across samples of the most common unique sequences clustered into (A) OTU-1 and (B) OTU-4. Colors are assigned to the top four most common unique sequences within each OTU and all remaining sequences from that OTU are assigned the color gray. Stacked bars in each position represent individual mice sampled for this study, and reflect the relative abundance of unique sequences in that sample. Mice are sorted by sites and then treatments, and finally by the total abundance of OTU-1. (PDF $49 \mathrm{~kb}$ )

Additional file 4: Expanded survival analysis. (PDF 44 kb)

\section{Abbreviations}

ACA: Acarbose; FDR: False-discovery rate; HPLC: High-performance liquid chromatography; ITP: Interventions testing program; LASSO: Least absolute shrinkage and selection operation; OTU: Operational taxonomic unit; PBS: Phosphate-buffered saline; S. alaskensis: Sphingopyxis alaskensis; SCFA: Short-chain fatty acid; TJL: The Jackson laboratory; UM: The University of Michigan; UT: The University of Texas Health Science Center at San Antonio

\section{Acknowledgments}

We would like to thank Marian Schmidt, Nicole Koropatkin, Anna Seekatz, Nielson Baxter, Clive Waldron, and members of the Schmidt Lab for feedback. We appreciate the technical support at each of the study sites for maintaining the animals and collecting the samples.

\section{Funding}

This research was supported by National Institute of Health, National Institute on Aging grants U01-AG022303 (RAM), U01-AG022308 (DEH), and

U01-AG022307 (RS), The Glenn Foundation for Medical Research (TMS), the Host Microbiome Initiative at the University of Michigan and an Integrated Training in Microbial Systems fellowship (BJS). An NIH NIA representative, as part of the U01 steering comittee, helped to plan the overarching experiment, but funding agencies were not involved in the collection, analysis, and interpretation of data presented here, nor in writing this manuscript.

\section{Availability of data and materials}

The sequence datasets generated and analyzed during the current study have been uploaded to the SRA database, accession SRP136736. Full-cohort survival data analyzed for portions of this study are available from the corresponding author on reasonable request. Code needed to reproduce data processing and analyses, raw HPLC data, and all metadata are available on GitHub [64].

\section{Authors' contributions}

$\mathrm{DEH}, \mathrm{RS}$, and RAM are principal ITP investigators at the three collaborating institutions and are responsible for design of the mouse experiment and supervision of technical personnel. ACE assembled and analyzed preliminary data. BJS collected the microbiome data and interpreted it along with TMS and RAM. BJS wrote a majority of the manuscript with contributions from TMS and RAM, who also helped with editing the manuscript. All authors read and approved the contents of the final article.

\section{Ethics approval and consent to participate}

All mice used in this study were maintained in specific-pathogen free conditions, and the protocols for husbandry and experimentation were approved by the Institutional Animal Care and Use Committees at each of the three institutions: reference numbers PRO00008565 at UM, 99084 at TJL, and 20040042AR at UT.

\section{Consent for publication}

Not applicable.

\section{Competing interests}

The authors declare that they have no competing interests. 


\section{Publisher's Note}

Springer Nature remains neutral with regard to jurisdictional claims in published maps and institutional affiliations.

\section{Author details}

${ }^{1}$ Department of Ecology \& Evolutionary Biology, University of Michigan, 48109 Ann Arbor, MI, USA. ${ }^{2}$ Department of Pathology and Geriatrics Center, University of Michigan, 48109 Ann Arbor, MI, USA. ${ }^{3}$ University of Missouri Metagenomics Center, University of Missouri, 65201 Columbia, MO USA. ${ }^{4}$ The Jackson Laboratory, 04609 Bar Harbor, ME USA. ${ }^{5}$ Department of Pharmacology, The University of Texas Health Science Center at San Antonio, 78229 San Antonio, TX, USA. ${ }^{6}$ Barshop Institute for Longevity and Aging Studies, 78245 San Antonio, TX USA. ${ }^{7}$ Geriatric Research, Education and Clinical Center and Research Service, South Texas Veterans Health Care System, 78229 San Antonio, TX USA. ${ }^{8}$ Department of Internal Medicine, University of Michigan, 48109 Ann Arbor, MI, USA

Received: 20 September 2018 Accepted: 17 May 2019

\section{Published online: 13 June 2019}

\section{References}

1. Nadon NL, Strong R, Miller RA, Harrison DE. NIA Interventions Testing Program: Investigating Putative Aging Intervention Agents in a Genetically Heterogeneous Mouse Model. EBioMedicine. 2017;21:3-4. https://doi.org/10.1016/j.ebiom.2016.11.038.

2. Harrison DE, Strong $\mathrm{R}$, Allison DB, Ames BN, Astle CM, Atamna H, Fernandez E, Flurkey K, Javors MA, Nadon NL, Nelson JF, Pletcher S, Simpkins JW, Smith DL, Wilkinson JE, Miller RA. Acarbose, 17- $\alpha$-estradiol, and nordihydroguaiaretic acid extend mouse lifespan preferentially in males. Aging Cell. 2014;13(2):273-82. https://doi.org/10.1111/acel.12170.

3. Strong R, Miller RA, Antebi A, Astle CM, Bogue M, Denzel MS, Fernandez E, Flurkey K, Hamilton KL, Lamming DW, Javors MA, de Magalhães JP, Martinez PA, McCord JM, Miller BF, Müller M, Nelson JF, Ndukum J, Rainger GE, Richardson A, Sabatini DM, Salmon AB, Simpkins JW, Steegenga WT, Nadon NL, Harrison DE. Longer lifespan in male mice treated with a weakly estrogenic agonist, an antioxidant, an $\alpha$-glucosidase inhibitor or a Nrf2-inducer. Aging Cell. 2016;15(5):872-84. https://doi.org/10.1111/acel.12496.

4. Harrison DE, Strong R, Alavez S, Astle CM, DiGiovanni J, Fernandez E, Flurkey K, Garratt M, Gelfond JAL, Javors MA, Levi M, Lithgow GJ, Macchiarini F, Nelson JF, Sukoff Rizzo SJ, Slaga TJ, Stearns T, Wilkinson JE, Miller RA. Acarbose improves health and lifespan in aging HET3 mice. Aging Cell. 2019;18(2):12898. https://doi.org/10.1111/acel.12898.

5. Laube H. Acarbose: An update of its therapeutic use in diabetes treatment. Clin Drug Inv. 2002;22(3):141-56.

6. Hanefeld M., Cagatay M., Petrowitsch T., Neuser D., Petzinna D., Rupp M. Acarbose reduces the risk for myocardial infarction in type 2 diabetic patients: Meta-analysis of seven long-term studies. Eur Heart J. 2004;25(1): 10-6. https://doi.org/10.1016/S0195-668X(03)00468-8.

7. Dehghan-Kooshkghazi M., Mathers J. C. Starch digestion, large-bowel fermentation and intestinal mucosal cell proliferation in rats treated with the $\alpha$-glucosidase inhibitor acarbose. Br J Nutr. 2004;91(03):357. https:// doi.org/10.1079/BJN20031063.

8. Weaver G. A., Tangel C. T., Krause J. A., Parfitt M. M., Jenkins P. L., Rader J. M., Lewis B. A., Miller T. L., Wolin M. J. Acarbose Enhances Human Colonic Butyrate Production. J Nutr. 1997;127(5):717-23. https://doi.org/10.1093/ jn/127.5.717.

9. Hiele M., Ghoos Y., Rutgeerts P., Vantrappen G. Effects of acarbose on starch hydrolysis. Dig Dis Sci. 1992;37(7):1057-64. https://doi.org/10. 1007/BF01300287.

10. Seifarth C., Bergmann J., Holst J. J., Ritzel R., Schmiegel W., Nauck M. A Prolonged and enhanced secretion of glucagon-like peptide 1 (7-36 amide) after oral sucrose due to $\alpha$-glucosidase inhibition (acarbose) in Type 2 diabetic patients. Diabet Med. 1998;15(6):485-91. https://doi.org/ 10.1002/(SICI)1096-9136(199806)15:6<485::AID-DIA610>3.0.CO;2-Y.

11. Qualmann C., Nauck M. A., Holst J. J., Orskov C., Creutzfeldt W. Glucagon-like peptide 1 (7-36 amide) secretion in response to luminal sucrose from the upper and lower gut. A study using $\alpha$-glucosidase inhibition (acarbose),. Scand J Gastroenterol. 1995;30(9):892-6. https:// doi.org/10.3109/00365529509101597.
12. Jenkins D. J. A., Taylor R. H., GoffD. V., Fielden H., Misiewicz J. J., Sarson D. L. Bloom S. R., Alberti K. G. M. M. Scope and specificity of acarbose in slowing carbohydrate absorption in man,. Diabetes. 1981;30(11):951-4. https://doi.org/10.2337/DIAB.30.11.951.

13. Weaver G. A., Tangel C. T., Krause J. A., Parfitt M. M., Stragand J. J., Jenkins P. L., Erb T. A., Davidson R. H., Alpern H. D., Guiney W. B., Higgins P. J. Biomarkers of human colonic cell growth are influenced differently by a history of colonic neoplasia and the consumption of acarbose. J Nutr. 2000;130(11): 2718-25. https://doi.org/10.1093/jn/130.11.2718.

14. Holt P. R., Atillasoy E., Lindenbaum J., Ho S. B., Lupton J. R., McMahon D., Moss S. F. Effects of acarbose on fecal nutrients, colonic $\mathrm{pH}$, and short-chain fatty acids and rectal proliferative indices. Metab: Clin Exp. 1996;45(9):1179-1187. https://doi.org/10.1016/S0026-0495(96)90020-7.

15. Wolever T. M. S., Chiasson J. L. Acarbose raises serum butyrate in human subjects with impaired glucose tolerance,. Br J Nutr. 2000;84(1):57-61. https://doi.org/10.1017/S0007114500001239.

16. Wolin M. J., Miller T. L., Yerry S., Bank S., Weaver G. A., Zhang Y. Changes of Fermentation Pathways of Fecal Microbial Communities Associated with a Drug Treatment That Increases Dietary Starch in the Human Colon Changes of Fermentation Pathways of Fecal Microbial Communities Associated with a Drug Treatment That Increas. Appl Environ Microbiol. 1999;65(7):2807-12

17. Zhang X., Fang Z., Zhang C., Xia H., Jie Z., Han X., Chen Y., Ji L. Effects of Acarbose on the Gut Microbiota of prediabetic patients: a randomized, Double-blind, controlled crossover trial,. Diabet Ther. 2017;8(2):293-307. https://doi.org/10.1007/s13300-017-0226-y.

18. Zhao L., Zhang F., Ding X., Wu G., Lam Y. Y., Shi Y., Shen Q., Dong W., Liu R., Ling Y., Zeng Y. Gut bacteria selectively promoted by dietary fibers alleviate type 2 diabetes. Science. 2018;1156(March):1151-6. https://doi. org/10.1126/science.aao5774.

19. Koh A., De Vadder F., Kovatcheva-Datchary P., Bäckhed F. From dietary fiber to host physiology: Short-chain fatty acids as key bacterial metabolites. Cell. 2016;165(6):1332-45. https://doi.org/10.1016/j.cell. 2016.05.041.

20. Kasubuchi M., Hasegawa S., Hiramatsu T., Ichimura A., Kimura I. Dietary gut microbial metabolites, short-chain fatty acids, and host metabolic regulation. Nutrients. 2015;7(4):2839-49. https://doi.org/10.3390/ nu7042839.

21. Wolever T. M. S., Fernandes J., Rao A. V. Serum Acetate:Propionate Ratio Is Related to Serum Cholesterol in Men but Not Women. J Nutr. 1996;126(11):2790-7. https://doi.org/10.1093/jn/126.11.2790.

22. Lagkouvardos I., Pukall R., Abt B., Foesel B. U., Meier-Kolthoff J. P., Kumar N. Bresciani A., Martínez I., Just S., Ziegler C., Brugiroux S., Garzetti D., Wenning M., Bui T. P. N., Wang J., HugenholtzF., Plugge C. M., Peterson D. A. Hornef M. W., Baines J. F., Smidt H., Walter J., Kristiansen K., Nielsen H. B., Haller D. Overmann J., Stecher B, Clavel T. The mouse intestinal bacterial collection (miBC) provides host-specific insight into cultured diversity and functional potential of the gut microbiota. Nat Microbiol. 2016;1(August):16131. https://doi.org/10.1038/nmicrobiol.2016.131.

23. Baxter N. T., Lesniak N. A., Sinani H., Schloss P. D., Koropatkin N. M. The Glucoamylase Inhibitor Acarbose has a diet-dependent and reversible effect on the murine gut Microbiome. mSphere. 2019;4(1):1-12. https:// doi.org/10.1128/msphere.00528-18.

24. Santilli A. D., Dawson E. M., Whitehead K. J., Whitehead D. C. Nonmicrobicidal small molecule inhibition of polysaccharide metabolism in human gut microbes: a potential Therapeutic Avenue. ACS Chem Biol. 2018;13(5):1165-72. https://doi.org/10.1021/acschembio.8b00309.

25. Lowe P. P., Gyongyosi B., Satishchandran A., Iracheta-Vellve A., Ambade A., Kodys K., Catalano D., Ward D. V., Szabo G. Alcohol-related changes in the intestinal icrobiome influence neutrophil infiltration, inflammation and steatosis in early alcoholic hepatitis in mice. PLOS ONE. 2017:12(3): 1-16. https://doi.org/10.1371/journal.pone.0174544.

26. Castoldi A., Andrade-Oliveira V., Aguiar C. F., Amano M. T., Lee J., Miyagi M. T., Latância M. T., Braga T. T., da Silva M. B., Ignácio A., Carola Correia Lima J. D., Loures F. V., Albuquerque J. A. T., Macêdo M. B., Almeida R. R., Gaiarsa J. W. Luévano-Martínez L. A., Belchior T., Hiyane M. I., Brown G. D., Mori M. A., Hoffmann C., Seelaender M., Festuccia W. T., Moraes-Vieira P. M., Câmara N. O. S. Dectin-1 activation exacerbates obesity and insulin resistance in the absence of MyD88. Cell Rep. 2017;19(11):2272-88. https://doi.org/10. 1016/j.celrep.2017.05.059. 
27. Singer B. H., Dickson R. P., Denstaedt S. J., Newstead M. W., Kim K., Falkowski N. R., Erb-Downward J. R., Schmidt T. M., Huffnagle G. B., Standiford T. J. Bacterial Dissemination to the Brain in Sepsis. Am J Respir Crit Care Med. 2018;197(6):747-6. https://doi.org/10.1164/rccm.201708$15590 \mathrm{C}$.

28. Ormerod K. L., Wood D. L. A., Lachner N., Gellatly S. L., Daly J. N., Parsons J. D., Dal'Molin C. G. O., Palfreyman R. W., Nielsen L. K., Cooper M. A., Morrison M., Hansbro P. M., Hugenholtz P. Genomic characterization of the uncultured Bacteroidales family S24-7 inhabiting the guts of homeothermic animals, Microbiome. 2016;4(1):36. https://doi.org/10.1186/s40168-016-0181-2.

29. Cole J.R., Wang Q., Fish J. A., Chai B., McGarrell D. M., Sun Y., Brown C. T., Porras-Alfaro A., Kuske C. R., Tiedje J. M. Ribosomal database project: data and tools for high throughput rRNA analysis,. Nucleic Acid Res. 2014;42(Database issue):633-42. https://doi.org/10.1093/nar/gkt1244.

30. Clavel T., Lagkouvardos I., Blaut M., Stecher B. The mouse gut microbiome revisited: From complex diversity to model ecosystems. Int J Med Microbiol. 2016;306(5):316-27. https://doi.org/10.1016/j.ijmm.2016. 03.002.

31. Lagkouvardos I., Lesker T. R., Hitch T. C. A., Gálvez E. J. C., Smit N., Neuhaus K., Wang J., Baines J. F., Abt B., Stecher B., Overmann J., Strowig T., Clavel T. Sequence and cultivation study of Muribaculaceae reveals novel species, host preference, and functional potential of this yet undescribed family. Microbiome. 2019;7(1):1-15. https://doi.org/10.1186/ s40168-019-0637-2.

32. Salzman N. H., de Jong H., Paterson Y., Harmsen H. J. M., Welling G. W. Bos N. A. Analysis of $16 \mathrm{~S}$ libraries of mouse gastrointestinal microflora reveals a large new group of mouse intestinal bacteria. Microbiology. 2002;148(11):3651-60. https://doi.org/10.1099/00221287-148-11-3651.

33. Wexler H. M. Bacteroides: The good, the bad, and the nitty-gritty. Clin Microbiol Rev. 2007;20(4):593-621. https://doi.org/10.1128/CMR.00008-07

34. Song Y., Liu C., Finegold S. M. Bacteroides. In: Whitman W. B., editor. Bergey's Manual of Systematics of Archaea and Bacteria. Hoboken, NJ: Wiley; 2015. https://doi.org/10.1002/9781118960608.gbm00238.

35. Macy J. M., Ljungdahl L. G., Gottschalk G. Pathway of succinate and propionate formation in Bacteroides fragilis. J Bacteriol. 1978;134(1):84-91.

36. Macfarlane S., Macfarlane G. T. Regulation of short-chain fatty acid production. Proc Nutr Soc. 2003;62(1):67-72. https://doi.org/10.1079/ PNS2002207.

37. Weaver G. A., Krause J. A., Miller T. L., Wolin M. J. Cornstarch fermentation by the colonic microbial community yields more butyrate than does cabbage fiber fermentation; cornstarch fermentation rates correlate negatively with methanogenesis. Am J Clin Nutr. 1992;55(1):70-77. https://doi.org/10.1093/ajcn/55.1.70.

38. Cummings J. H., Englyst $H$. N. Fermentation in the human large intestine and the available substrates. Am J Clin Nutr. 1987;45:1243-55.

39. Wang S., Huang M., You X., Zhao J., Chen L., Wang L., Luo Y., Chen Y. Gut microbiota mediates the anti-obesity effect of calorie restriction in mice. Sci Rep. 2018;8(1):2-15. https://doi.org/10.1038/s41598-018-313531. arXiv:1008.3864v1.

40. Zhang C., Li S., Yang L., Huang P., Li W., Wang S., Zhao G., Zhang M., Pang X., Yan Z., Liu Y., Zhao L. Structural modulation of gut microbiota in life-long calorie-restricted mice. Nat Commun. 2013;4:1-10. https://doi. org/10.1038/ncomms3163. arXiv:1011.1669v3.

41. Maslowski K. M., Vieira A. T., Ng A., Kranich J., Sierro F., Di Yu, Schilter H. C. Rolph M. S., MacKay F., Artis D., Xavier R. J., Teixeira M. M., MacKay C. R. Regulation of inflammatory responses by gut microbiota and chemoattractant receptor GPR43. Nature. 2009;461(7268):1282-6. https:// doi.org/10.1038/nature08530. NIHMS150003.

42. Smith P. M., Howitt M. R., Panikov N., Michaud M., Ann C., Bohlooly-y M., Glickman J. N., Garrett W. S. The Micorbial metabolites, short chain fatty acids, regulate Treg cell homeostasis. Science. 2013;341(6145):569-74. https://doi.org/10.1126/science.1241165.The.

43. Samuel B. S., Shaito A., Motoike T., Rey F. E., Backhed F., Manchester J. K. Hammer R. E., Williams S. C., Crowley J., Yanagisawa M., Gordon J. I. Effects of the gut microbiota on host adiposity are modulated by the short-chain fatty-acid binding G protein-coupled receptor, Gpr41,. Proc Natl Acad Sci of the U S A. 2008;105(43):16767-72. https://doi.org/10. 1073/pnas.0808567105.

44. Byrne C. S., Chambers E. S., Morrison D. J., Frost G. The role of short chain fatty acids in appetite regulation and energy homeostasis. Int J Obes. 2015;39(9):1331-8. https://doi.org/10.1038/ijo.2015.84.
45. Venter C. S., Vorster H. H., Cummings J. H. Effects of dietary propionate on carbohydrate and lipid metabolism in healthy volunteers, Am J Gastroenterol. 1990;85(5):549-53.

46. Waldecker M., Kautenburger T., Daumann H., Busch C., Schrenk D. Inhibition of histone-deacetylase activity by short-chain fatty acids and some polyphenol metabolites formed in the colon. J Nutr Biochem. 2008;19(9):587-93. https://doi.org/10.1016/j.jnutbio.2007.08.002.

47. Adcock I. M. HDAC inhibitors as anti-inflammatory agents. Br J Pharmacol 2007;150(7):829-31. https://doi.org/10.1038/sj.bjp.0707166.

48. Johnstone R. W. Histone-deacetylase inhibitors: novel drugs for the treatment of cancer. Nat Rev Drug Discov. 2002;1(4):287-99. https://doi. org/10.1038/nrd772.

49. Mistry R. H., Gu F., Schols H. A., Verkade H. J., Tietge U. J. F. Effect of the prebiotic fiber inulin on cholesterol metabolism in wildtype mice. Sci Rep. 2018;8(1):1-8. https://doi.org/10.1038/s41598-018-31698-7.

50. Rozan P., Nejdi A., Hidalgo S., Bisson J.-F., Desor D., Messaoudi M. Effects of lifelong intervention with an oligofructose-enriched inulin in rats on general health and lifespan. Br J Nutr. 2008;100(06):1192. https://doi.org/ 10.1017/S0007114508975607.

51. Matsumoto M., Kurihara S., Kibe R., Ashida H., Benno Y. Longevity in mice is promoted by probiotic-induced suppression of colonic senescence dependent on upregulation of gut bacterial polyamine production. PLoS ONE. 2011;6(8). https://doi.org/10.1371/journal.pone.0023652.

52. McGonigle P., Ruggeri B. Animal models of human disease: Challenges in enabling translation. Biochem Pharmacol. 2014;87(1):162-71. https://doi. org/10.1016/j.bcp.2013.08.006.

53. Mobley A., Linder S. K., Braeuer R., Ellis L. M., Zwelling L. A Survey on Data Reproducibility in Cancer Research Provides Insights into Our Limited Ability to Translate Findings from the Laboratory to the Clinic. PLoS ONE. 2013;8(5):3-6. https://doi.org/10.1371/journal.pone.0063221.

54. Laukens D., Brinkman B. M., Raes J., De Vos M., Vandenabeele P. Heterogeneity of the gut microbiome in mice: Guidelines for optimizing experimental design. FEMS Microbiology Reviews. 2015;40(1):117-32. https://doi.org/10.1093/femsre/fuv036.

55. Stappenbeck T. S., Virgin H. W. Accounting for reciprocal host-microbiome interactions in experimental science. Nature. 2016;534(7606):191-9. https://doi.org/10.1038/nature18285.

56. Miller R. A., Harrison D. E., Astle C. M., Fernandez E., Flurkey K., Han M., Javors M. A., Li X., Nadon N. L., Nelson J. F., Pletcher S., Salmon A. B. Sharp Z. D., Van Roekel S., Winkleman L., Strong R. Rapamycin-mediated lifespan increase in mice is dose and sex dependent and metabolically distinct from dietary restriction. Aging Cell. 2014;13(3):468-77. https://doi org/10.1111/acel.12194.

57. Morrison D. J., Preston T. Formation of short chain fatty acids by the gut microbiota and their impact on human metabolism. Gut Microbes. 2016;7(3):189-200. https://doi.org/10.1080/19490976.2015.1134082.

58. Canfora E. E., Jocken J. W., Blaak E. E. Short-chain fatty acids in control of body weight and insulin sensitivity. Nat Rev Endocrinol. 2015;11(10): 577-91. https://doi.org/10.1038/nrendo.2015.128.

59. Miller R. A., Harrison D. E., Astle C. M., Baur J. A., Boyd A. R., de Cabo R., Fernandez E., Flurkey K., Javors M. a., Nelson J. F., Orihuela C. J., Pletcher S. Sharp Z. D., Sinclair D. A., Starnes J. W., Wilkinson J. E., Nadon N. L., Strong R. Rapamycin, but not resveratrol or simvastatin, extends life span of genetically heterogeneous mice. J Gerontol A Biol Sci Med Sci. 2011;66 A(2):191-201. https://doi.org/10.1093/gerona/glq178.

60. Smets W., Leff J. W., Bradford M. A., McCulley R. L., Lebeer S., Fierer N. A method for simultaneous measurement of soil bacterial abundances and community composition via $16 \mathrm{~S}$ rRNA gene sequencing. Soil Biol Biochem. 2016;96:145-51. https://doi.org/10.1016/j.soilbio.2016.02.003.

61. Stämmler F., Gläsner J., Hiergeist A., Holler E., Weber D., Oefner P. J., Gessner A., Spang R. Adjusting microbiome profiles for differences in microbial load by spike-in bacteria. Microbiome. 20161-13. https://doi. org/10.1186/s40168-016-0175-0.

62. Kozich J. J., Westcott S. L., Baxter N. T., Highlander S. K., Schloss P. D. Development of a dual-index sequencing strategy and curation pipeline for analyzing amplicon sequence data on the miseq illumina sequencing platform. Appl Environ Microbiol. 2013;79(17):5112-20. https://doi.org/10. 1128/AEM.01043-13.

63. Schloss P. D., Westcott S. L., Ryabin T., Hall J. R., Hartmann M., Hollister E. B., Lesniewski R. A., Oakley B. B., Parks D. H., Robinson C. J., Sahl J. W., Stres B., Thallinger G. G., Van Horn D. J., Weber C. F. Introducing mothur: 
open-source, platform-independent, community-supported software for describing and comparing microbial communities. Appl Environ Microbiol. 2009;75(23):7537-41. https://doi.org/10.1128/AEM.01541-09.

64. Smith B. J. Code and Metadata to Reproduce: Changes in the gut microbiota and fermentation products associated with enhanced longevity in acarbose-treated mice. 2018. https://doi.org/10.5281/ zenodo.1229203. https://github.com/bsmith89/smith2019paper/releases Accessed 25 Apr 2018.

65. Schloss P. D. Silva reference files. 2018. https://www.mothur.org/wiki/ Silva_reference_files. Accessed 1 Feb 2018.

66. Wang Q., Garrity G. M., Tiedje J. M., Cole J. R. Naïve Bayesian classifier for rapid assignment of rRNA sequences into the new bacterial taxonomy. Appl Environ Microbiol. 2007;73(16):5261-7. https://doi.org/10.1128/AEM. 00062-07. Wang, Qiong, 2007, Naive.

67. Yilmaz P., Parfrey L. W., Yarza P., Gerken J., Pruesse E., Quast C., Schweer T., Peplies J., Ludwig W., Glöckner F. O. The SILVA and "all-species Living Tree Project (LTP)" taxonomic frameworks. Nucleic Acids Res. 2014;42(D1): 643-8. https://doi.org/10.1093/nar/gkt1209.

68. Westcott S. L., Schloss P. D. OptiClust, an Improved Method for Assigning Amplicon-Based Sequence Data to Operational Taxonomic Units. mSphere. 2017;2(2):00073-17. https://doi.org/10.1128/mSphereDirect. 00073-17.

69. Wheeler D. L., Chappey C., Lash A. E., Leipe D. D., Madden T. L., Shuler G. D., Tatusova T. A., Rapp B. A. Database resources of the National Center for Biotechnology Information,. Nucleic Acids Res. 2000;41 (November 2012): 8-20. https://doi.org/10.1093/nar/gks1189.

70. Oksanen J., Blanchet F. G., Friendly M., Kindt R., Legendre P., McGlinn D., Minchin P. R., O'Hara R. B., Simpson G. L., Solymos P., Stevens M. H. H., Szoecs E., Wagner H. vegan: Community Ecology Package. 2018. https:// cran.r-project.org/web/packages/vegan. Accessed 24 May.

71. Love M. I., Huber W., Anders S. Moderated estimation of fold change and dispersion for RNA-seq data with DESeq2. Genome Biol. 2014;15(12):550. https://doi.org/10.1186/s13059-014-0550-8.

72. Benjamini Y., Hochberg Y. J R Stat Soc Ser B Stat Methodol. 1995;57(1): 289-300.

73. Pedregosa F., Varoquaux G., Gramfort A., Michel V., Thirion B., Grisel O., Blondel M., Prettenhofer P., Weiss R., Dubourg V., Vanderplas J., Passos A., Cournapeau D., Brucher M., Perrot M., Duchesnay É,. Scikit-learn: Machine Learning in Python. J Mach Learn Res. 2011;12:2825-30. http:// arxiv.org/abs/1201.0490

74. Iman R. L., Conover W. J. Approximations of the critical region for spearman's rho with and without ties present. Commun Stat Simul Comput. 1978;7(3):269-82. https://doi.org/10.1080/03610917808812076.

75. Therneau T. M. A Package for Survival Analysis in S. 2015. https://cran.rproject.org/web/packages/survival. Accessed 24 May.

Ready to submit your research? Choose BMC and benefit from:

- fast, convenient online submission

- thorough peer review by experienced researchers in your field

- rapid publication on acceptance

- support for research data, including large and complex data types

- gold Open Access which fosters wider collaboration and increased citations

- maximum visibility for your research: over 100M website views per year

At $\mathrm{BMC}$, research is always in progress.

Learn more biomedcentral.com/submissions 